\title{
Article \\ Interpretation of Nondestructive Magnetic Measurements on Irradiated Reactor Steel Material
}

\author{
Gábor Vértesy ${ }^{1, *}$, Antal Gasparics ${ }^{1}\left[\mathbb{C}\right.$, Ildikó Szenthe ${ }^{1}$ and Inge Uytdenhouwen ${ }^{2}$ \\ 1 Centre for Energy Research, 1121 Budapest, Hungary; gasparics.antal@energia.mta.hu (A.G.); \\ szenthe.ildiko@energia.mta.hu (I.S.) \\ 2 SCK CEN Belgian Nuclear Research Centre, 2400 Mol, Belgium; inge.uytdenhouwen@sckcen.be \\ * Correspondence: gabor.vertesy@energia.mta.hu
}

Citation: Vértesy, G.; Gasparics, A.; Szenthe, I.; Uytdenhouwen, I. Interpretation of Nondestructive Magnetic Measurements on Irradiated Reactor Steel Material. Appl. Sci. 2021, 11, 3650. https:// doi.org/10.3390/app11083650

Academic Editors: Stefano Invernizzi and Giuseppe Lacidogna

Received: 8 March 2021

Accepted: 15 April 2021

Published: 18 April 2021

Publisher's Note: MDPI stays neutral with regard to jurisdictional claims in published maps and institutional affiliations.

Copyright: (c) 2021 by the authors. Licensee MDPI, Basel, Switzerland. This article is an open access article distributed under the terms and conditions of the Creative Commons Attribution (CC BY) license (https:// creativecommons.org/licenses/by/ $4.0 /)$.
Featured Application: In this paper, the application of a nondestructive magnetic testing method is analyzed for neutron irradiation-generated embrittlement of nuclear pressure vessel materials. It was found that in spite of the big scatter of measurement points, the degradation of reactor pressure vessel steel can be successfully predicted by this technique. Furthermore, it turned out that the embrittlement highly depends on the local material conditions, which was found to be very different even within the same block of reactor steel material. This implicates different trajectories of their neutron irradiation induced degradation, as well as identifiable differences in the outcomes of their nondestructive evaluation. The magnetic descriptor results add reliable and precise determination of local embrittlement in the material than the traditionally used destructive mechanical parameters, so the results can be helpful for the future practical application of this technique for regular inspection of nuclear reactors.

Abstract: Neutron irradiation-generated embrittlement of nuclear pressure vessel steel was inspected by a nondestructive magnetic method, called magnetic adaptive testing (MAT). This method is based on systematic measurement and evaluation of minor magnetic hysteresis loops. Result of MAT measurement was compared with the result of the traditional Charpy measurement. Good correlation was found between these parameters. One of the main findings of the present work is that the considerable part of scatter of points obtained by magnetic measurement can be attributed to local material inhomogeneity. Another important conclusion is that the embrittlement highly depends on the initial local material conditions, i.e., the initial microstructure, which are very different even within the same block of reactor steel material. By taking this into account, the magnetic descriptors obtain more precise determination of the local embrittlement than the traditionally used destructive mechanical parameters from Charpy data.

Keywords: neutron irradiation embrittlement; reactor pressure vessel; magnetic nondestructive evaluation; magnetic adaptive testing; ductile to brittle transition temperature

\section{Introduction}

Nuclear power plants (NPPs) play an important role in the energy production. Their safety is a very important aspect and because of this the inspection of their integrity is a permanent task. Some of them are going to the end of their predicted lifetime. In many countries, the extension of lifetime of existing NPPs has been accepted. This strategy ensures adequate supply of electric energy in the near future. However, reliable tools are necessary for estimating the remaining lifetime of NPP components to ensure their long-term operation. The reactor pressure vessel (RPV) is the most important, irreplaceable part of NPPs, which determines the possibility of long-term operation. The main ageing process of RPVs is the irradiation embrittlement and it is the main life limiting degradation of the pressurized and boiling water reactors. The secure lifetime of an operation depends 
on several factors, such as chemical composition, design, mechanical and microstructural properties of the RPV steels, operation conditions, defect occurrence, and so on [1].

This irradiation embrittlement, generated by long-term and high-energy neutrons, causes mechanical property changes: increase of hardness, yield stress, and tensile strength, and also decrease of toughness [2]. However, testing and the evaluation of radiation embrittlement is a difficult task. The radiation embrittlement is not one simple degradation mechanism, but the sum of several quite different processes, such as (i) direct matrix damage due to neutron bombardment (increase of the dislocation density), (ii) precipitation hardening the matrix ( $\mathrm{Cu}$ is the leading element, but nickel, manganese, silicon, etc. also have an influence) and (iii) segregation ( $\mathrm{P}$ is a recognized segregating element) and if $P$ covers the grain boundary even only in one atom thickness, it can cause non-hardening embrittlement.

In the second generation of the NPPs, the so-called surveillance specimens that are located inside of the vesseland are withdrawn after certain periods and destructively tested. The traditional evaluation of the embrittlement is the mechanical testing: tensile, Charpy impact, fracture toughness, and hardness testing [3]. Effort were made to apply nondestructive methods to measure the rate of the embrittlement, for instance measurement of the Seebeck coefficient [4] or application of the well-known ultrasonic technique [5]. Considering that most of the presently operating nuclear reactors are Pressurized Water Reactors (PWRs) and a RPV is made from low alloyed steels, magnetic methods (hysteresis measurements, Barkhausen noise measurement, magnetoacoustics emission) can be successfully applied on them. Ref. [6] gives a general overview about the application possibility of magnetic methods in nondestructive testing. Magnetic tests are also considered as a potential NDE method to measure radiation embrittlement. It is theoretically and experimentally known that several magnetic properties are sensitive to microstructure [7]. The radiation embrittlement is usually measured by a shift of the energy-temperature curves obtained by impact testing of Charpy V-notch specimens. This shift increases the brittle-to-ductile transition temperature, and is generally followed by hardening; the hardness, yield, and ultimate tensile strength increases and the elongation decreases. The magnetic Barkhausen noise method can also be used to determine the irradiation effects on nuclear reactor structural materials. In Ref. [8], Barkhausen emission results were correlated to neutron fluence and overall hardening was observed on all materials exposed to irradiation.

There also exists a nondestructive micromagnetic method, which can also be successfully applied for the inspection of the damage in ferromagnetic RPV steels. This is the so-called Micromagnetic Multiparameter Microstructure and Stress Analysis (3MA), which combines several methods [9]. These nondestructive methods for characterizing materials properties are based on physical principle that magnetic behaviour is correlated to macroscopic physical properties of materials [10]. However, the first step of any practical application of nondestructive techniques should be to find a correlation between them and destructively measured, standardized parameters. It means that a rigorous calibration is necessary before any nondestructive technology can be validated.

In several of our recent papers [11-13], the neutron irradiation-generated embrittlement of RPV steels was studied by a novel magnetic nondestructive testing method, called magnetic adaptive testing, MAT $[14,15]$. The MAT method is based on minor magnetic hysteresis loops measurement and it has a lot of advantages compared with the traditional magnetic hysteresis measurements. In these works, the destructively determined ductile-to-brittle transition temperature (DBTT) values were compared with the magnetic parameters, measured nondestructively by measuring minor magnetic hysteresis loops on reactor pressure vessel materials irradiated by different neutron doses. At any time, a definite, significant correlation between DBTT and MAT parameters was found.

In a very recent paper by us [13], four types of RPV steels (three base metals and one weld material) were studied by MAT and also by other magnetic methods. Beyond the confirmation of the above-mentioned correlation between magnetic parameters and material hardening, a large scatter of points was also observed, but the correlation between 
MAT descriptors and transition temperature was clearly seen and the obtained correlation could be used for the estimation of the real DBTT values. This correlation is shown in Figure 1. It is evident that the points scatter significantly. This scatter was "partly" attributed to the different surface conditions of the investigated specimens [13]. However, it is suspected that the surface condition is not the only reason of this big scatter: different material properties of measured samples can also play an important role. The purpose of the present work is to analyze this other possible reason for this scatter.

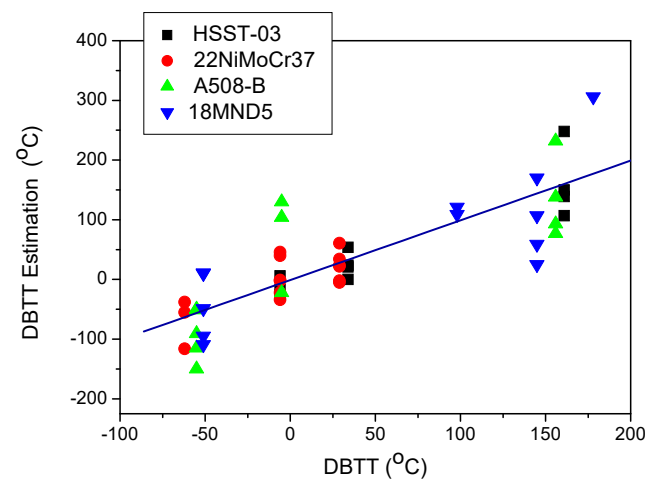

Figure 1. Estimated DBTT values as functions of the measured DBTT values for the investigated three base metals, 22NiMoCr37, A508-B, and HSST-03, and for one weld metal, 18MND5-W [11].

A series of measurements were carried out on another type of RPV steel (15Kh2NMFA), before and after neutron irradiation, and the result of the MAT measurements were compared with the destructively determined transition temperature. Standard Charpy specimens were used for investigation. For checking the conclusion of the above-mentioned previous work, a special care was made to produce specimens with exactly the same quality of surface to reduce the possible influence of the surface roughness on the measured magnetic quantities. A detailed analysis of the error of MAT descriptors is also presented in the Appendix A.

\section{Materials and Methods}

\subsection{Materials}

In our investigations, the so-called $\mathrm{Cr}-\mathrm{Ni}-\mathrm{MoV}$ steel RPV material was used. The 15Kh2NMFA forging steel is manufactured by the Russian IZHORA company for a 1000MW WWER reactor. The original heat number of it is 181,358, produced according to the Russian specification TU 108.765-78. The chemical composition is given in Table 1. The as-received specimens have a mixed tempered ferrite-bainite microstructure.

Table 1. Chemical composition (wt.\%) of the 15Kh2NMFA material.

\begin{tabular}{cccccccccc}
\hline $\mathbf{C}$ & $\mathbf{M n}$ & $\mathbf{S i}$ & $\mathbf{S}$ & $\mathbf{P}$ & $\mathbf{C r}$ & $\mathbf{N i}$ & $\mathbf{M o}$ & $\mathbf{V}$ & $\mathbf{C u}$ \\
\hline 0.16 & 0.42 & 0.29 & 0.008 & 0.0012 & 1.97 & 1.29 & 0.52 & 0.12 & 0.12 \\
\hline
\end{tabular}

Charpy specimens (ISO-V) were cut out from the same vessel block at $\frac{3}{4}$ depth. Orientation of the specimens is selected as T-L according to ASTM E23-16b (Standard Test Methods for Notched Bar Impact Testing of Metallic Materials).

Altogether 13 pieces (Nos. 1 to 13) of the manufactured Charpy specimens were used for the present investigations. They were measured by the nondestructive MAT method before and after neutron irradiation. Specimens were irradiated by neutrons in the BR2 reactor at SCK CEN, at different irradiation fluences in the primary water pool. Temperature of irradiation was between 100 and $120 \mathrm{C}$. Neutron irradiation was done in a rig called NOMAD_3 [16]. This process ensured that the created damage is large enough to detect it by nondestructive methods. The fluence was between 1.55 and $7.90 \times 10^{19} \mathrm{n} / \mathrm{cm}^{2}$ 
(E $>1 \mathrm{MeV}$ ). Charpy impact testing standard (ASTM-23-16b, [17]) was used to get the $41 \mathrm{~J}$ ductile-to-brittle transition temperature (DBTT). A $300 \mathrm{~J}$ Toni MFL pendelum was used for testing with an instrumented ISO tup. All the tests were made according to the ASTM E23 and E2215 prevailing standards. The values of DBTT were determined for groups of specimens irradiated by the same neutron fluence. The error of DBTT usually does not exceed $+/-15{ }^{\circ} \mathrm{C}$, except in inhomogeneous materials.

\subsection{Magnetic Adaptive Testing}

Magnetic adaptive testing is a special way of magnetic hysteresis measurements. Instead of the major hysteresis loop, a series of minor loops are measured with step-by-step increasing amplitude of a triangular waveform magnetizing current. The variation of the magnetizing field is linear with time; consequently, the signal of the pick-up coil is proportional to the sample's differential permeability. The measured minor loops (permeability loops) serve as the input for the further evaluation of data. Each point of permeability loops represents some information about the magnetic behaviour of the sample. During the evaluation process, permeability matrices are calculated from permeability data, and each matrix element is compared with the corresponding element of the reference sample. The elements of permeability matrices are characterized by $\mu \equiv \mu\left(h_{a}, h_{b}\right)$ values. Here, $h_{a}$ is the magnetizing field and $h_{b}$ is the amplitude of minor loop. $\mu$-matrix elements are called as "MAT-descriptors." In this way, a big data pool is generated and these descriptors characterize magnetically the investigated material. They are considered as functions of any independent parameter, describing the degradation of the material due to any external influence. The purpose of the MAT evaluation is to choose from these data pools those $\mu$ $(x)$-degradation functions that best characterize the actual material degradation. $x$ is the DBTT transition temperature in the present work. The MAT procedure and evaluation is described in detail in [15].

The sensitivity and reliability of the chosen-so-called optimal MAT descriptors-is essential for the effective application of MAT. For checking these parameters (sensitivity and reliability), a "sensitivity map" of degradation functions is generated, which indicates relative sensitivity of degradation functions with respect to independent variable of the measured material. The degradation function's sensitivity is calculated as the slope of its linear regression. In the graph of the sensitivity map, it is expressed by a colour, giving useful information about the relative modification of the studied magnetic parameter with respect to the independent characteristic. The most sensitive MAT-descriptors are taken from the top area of "hills" of the elevated sensitivity values. The sensitivity map also indicates the reliability of the chosen descriptor. Big plateaus are favourable, where the parameters only slightly depend on the actual choice of $h_{a}$ and $h_{b}$ field coordinates values. See the sensitivity map in Appendix A.

The measured samples were magnetized by a yoke, which was put on the top of the sample, opposite to the V-notch. A half transformer core was used as magnetizing yoke. It is made of laminated Fe-Si sheets. Two coils, a pick-up coil and an exciting coil, are wound on yoke legs. The cross-section of the yoke leg was $10 \times 5 \mathrm{~mm}^{2}$, corresponding to the sample size. The sample holder was designed for usage in a hot cell, made possible by the replacement of the samples by a manipulator.

Analyzing carefully the possible error of those MAT descriptors that characterize the material degradation, it was found that the uncertainty of this parameter is less than $1 \%$. Considering the importance of MAT descriptors' uncertainty, a detailed analysis can be found in the Appendix A; they are the possible sources of this error, and the analysis shows how this uncertainty can be determined.

\section{Results}

Magnetic measurements and mechanical tests were performed on the same samples before and after neutron irradiaton. As has been reported presently [13], irradiaton caused measurable changes in the material properties. The correlation between the transition 
temperature and neutron fluence is not linear: relatively low neutron dosage causes a relatively large increase of $\triangle \mathrm{DBBT}$, as shown in Figure 2 (or $\Delta \mathrm{T}_{41 \mathrm{~J}}$ in the Figure), and later, a larger dosage causes smaller changes in material embrittlement due to the accumulation of the induced damages. The sample series 15Kh2NMFA in Figure 2 is the same as was measured in the present work.

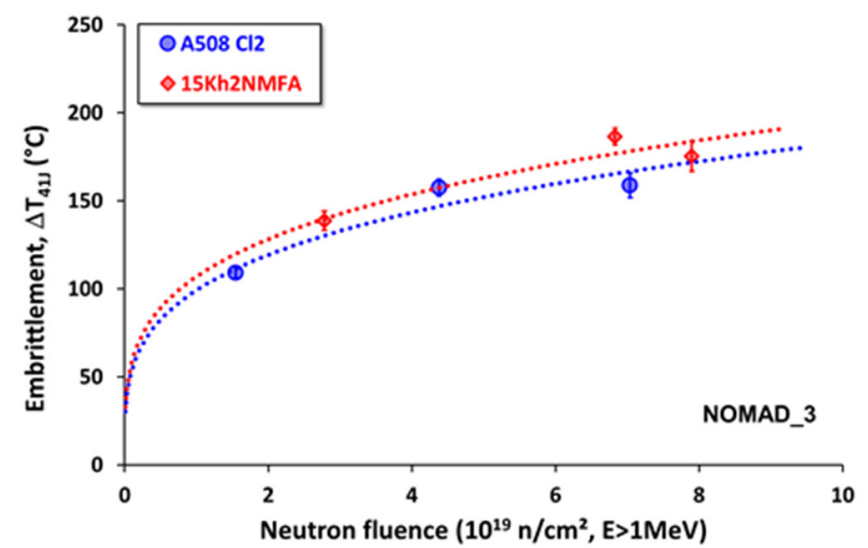

Figure 2. Embrittlement of RPV steel materials after neutron irradiation as a function of the neutron fluence. The dashed lines are added to guide the eye (power law fit) [13].

Good, monotonic correlation was found between nondestructively measured magnetic parameters (regardless on the used measurement technique) and mechanical hardening (determined by DBBT, Vickers hardness, and uniaxial tensile tests). However, a large scatter of magnetic parameters was found again, similarly as reported in [11]. To clarify this problem, the results of the MAT measurements on 15Kh2NMFA type material are analyzed in this section. The result of the MAT evaluation as function of the transition temperature is shown in Figure 3. "Optimally chosen MAT descriptor" is the parameter that describes in the most adequate way the embrittlement of the material due to neutron irradiation. In other words, this is the parameter that ensured the highest sensitivity at good reproducibility. In our case (plotted in Figure 3), this descriptor is characterized by $h_{a}=-30 \mathrm{~mA}$ and $h_{b}=1080 \mathrm{~mA}$ magnetic field values. The tendency of increasing embrittlement due to neutron irradiation is clearly seen, but the large scatter of points is also evident. In this figure, all measured results are plotted together, and the influence of neutron irradiation on individual samples is not seen.

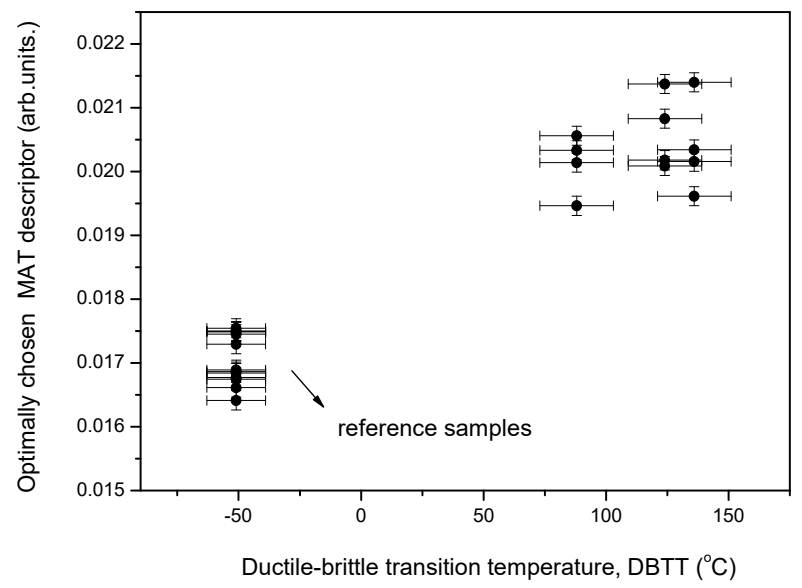

Figure 3. Optimally chosen MAT descriptors as a function of the transition temperature for the material 15Kh2NMFA. 
In spite of the fact that Charpy samples were cut from the same block of reactor steel material, they are magnetically rather different, even before irradiation, as can also be seen in Figure 3 (reference samples).

In order to study the magnetic behaviour that makes the difference between the samples more visible, the measured permeability loops of all not-irradiated (reference) samples are shown in Figure 4. In Figure 4a, total loops (including all minor loops as well) are shown, while the magnified part of the graph is shown in Figure $4 \mathrm{~b}$. In this latter case, only the envelope of the loops (minor loops with the largest amplitude) can be seen.
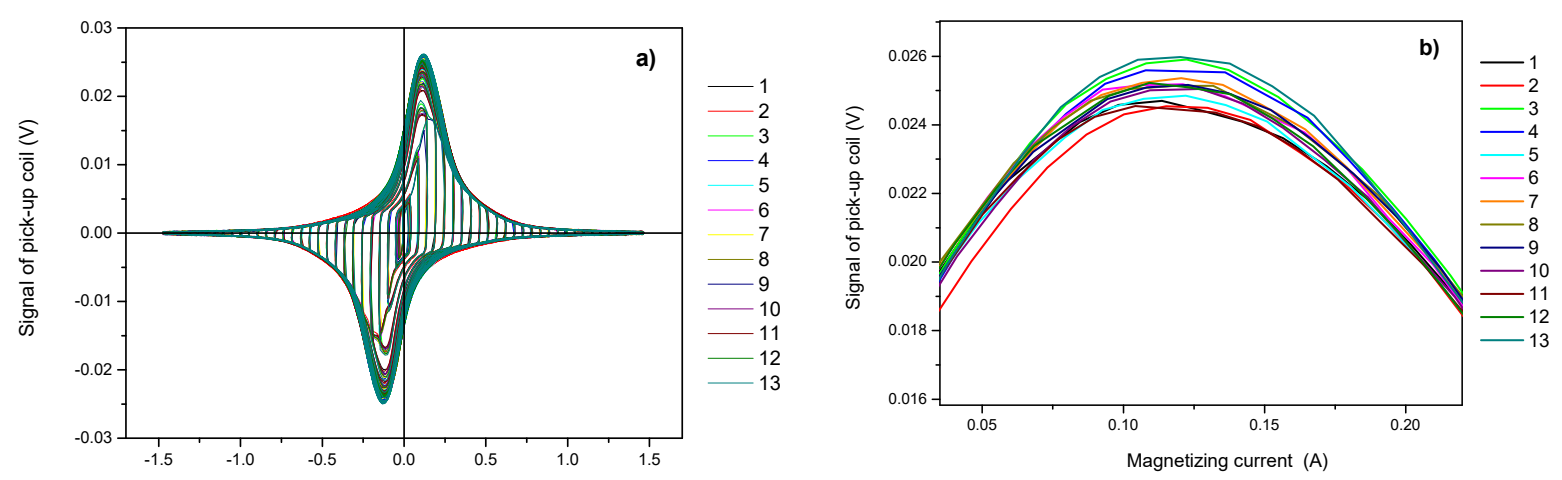

Figure 4. The measured permeability loops of the investigated, not-irradiated samples. (a) Full loops, (b) magnified parts of the loops.

Another way of plotting was used in Figure 5 to show the influence of the neutron irradiation on the individual samples. In this way of presentation, each point represents one sample, and the MAT descriptor is normalized in each case with the same descriptor measured on the same sample before irradiation. This is why only one point shows the reference case. Neutron irradiation caused a $10-24 \%$ increase of magnetic parameters compared with the same parameter of a virgin sample. Evidently, this way of plotting did not reduce the scatter of points. Nevertheless, the tendency is clearly seen. A more or less linear correlation was found between the transition temperature and the MAT parameter modification. The scatter of points is demonstrated also by the $\mathrm{R}=0.783$ regression factor of the linear fit.

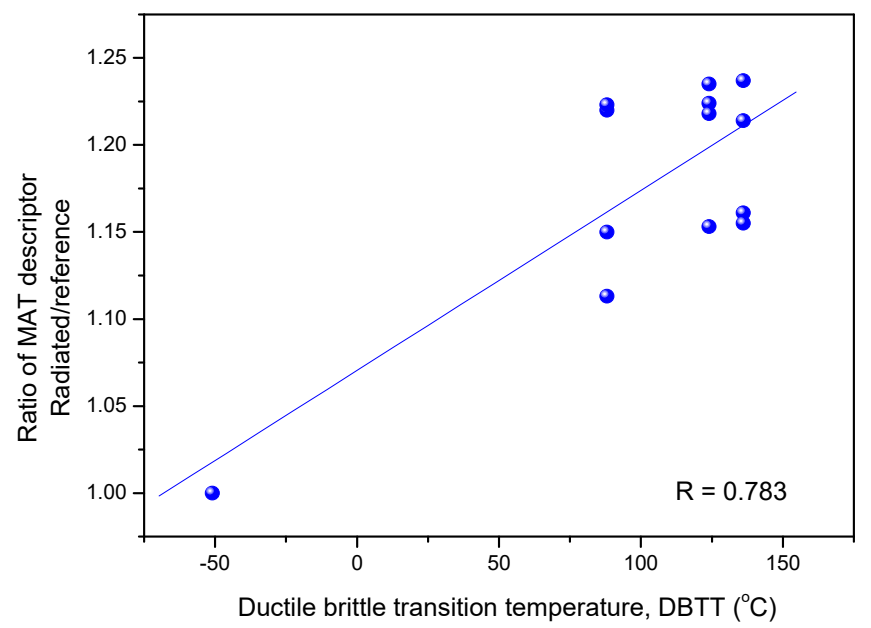

Figure 5. Ratio (normalized to the reference condition) of the optimally chosen MAT descriptor of all irradiated and not-irradiated samples as a function of the transition temperature.

The possible influence of the magnetic behaviour by the neutron irradiation-generated degradation of the reactor pressure vessel steel is shown in Figure 6. A subset of samples was chosen from the measured set of samples, which behave similarly from a magnetic 
point of view. These are samples 6, 7, 10, and 12; thus, four Charpy samples out of thirteen samples. This is shown in Figure $4 \mathrm{~b}$. The maximal permeability of these samples is in the middle region (indicated by orange, magenta, green, and violet colours). If only the magnetically similar samples are considered, the scatter of points (as seen in Figure 5) is dramatically reduced (see Figure 6). The regression of the linear fit increases to $R=0.996$.

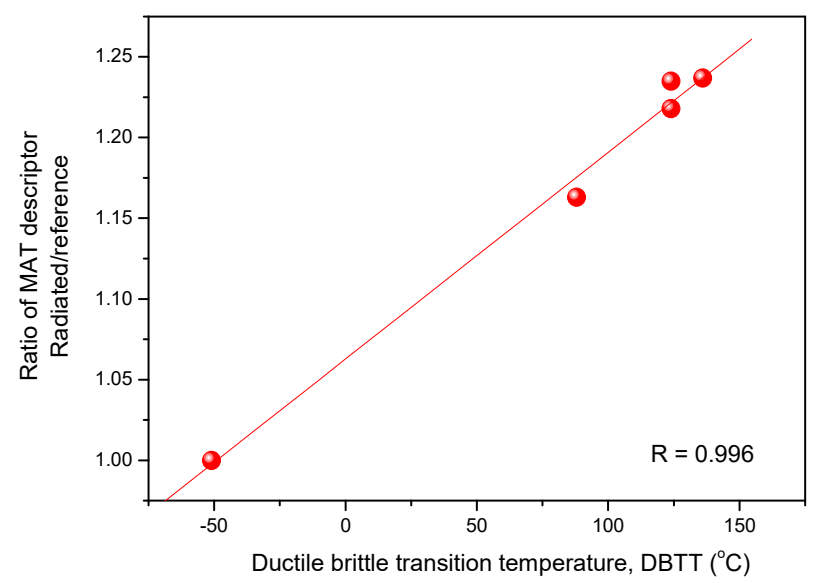

Figure 6. Ratio (normalized to the reference condition) of the optimally chosen MAT descriptor of selected irradiated and not-irradiated samples as a function of the transition temperature.

\section{Discussion}

Considering the above-described experiment and its interpretation, a possible and very probable explanation was found for the large scatter of magnetic parameters as functions of transition temperature, experienced in our previous works [11,13] and shown in Figures 3 and 5. It is not the error of the magnetic measurement itself, but it is originated in the inhomogeneity of the material, which results in different initial microstructural, as well as mechanical conditions resulting in different magnetic behaviours. It is an important observation that this inhomogeneity also exists for samples cut from the same block. The differences in the initial material conditions lead to different trajectories of the irradiation embrittlement, and to different final states, accordingly.

To clarify that we did not use backward reasoning to decide which data points fit best to our hypothesis, we shall briefly detail the process: (1) We observed a large scatter of MAT descriptors (see Figure 6) measured on the reference and irradiated samples. (2) Regardless of the result of the MAT evaluation, we compared the magnetic behaviour of reference samples, and it was found that there were four samples that behaved similarly from a magnetic point if view. (3) The MAT evaluation was repeated, taking into account only the selected samples. This selection did not give any information about the behaviour of the irradiated samples; by taking this selection, we did not know how the irradiated samples would behave.

Irradiation ageing, in practice, is the sum of the following mechanism: occurance of stable and unstable defects, precipitations, segregations, thermal ageing, and thermal recovery. The ratio of the effects of the different mechanisms depends not only on the alloy type (including the effect of polluting elements), the irradiation fluence, flux, and temperature, but also on the initial local microstructure state, as can be interpreted from our experiments. The destructively measured mechanical parameters were measured on a set of samples but not individually, in contrast to the magnetic measurements. DBTT always involves multiple specimens; it cannot be derived from one single specimen. This is the reason for the large error of the DBTT values, as shown in Figure 3. Magnetic measurements characterize better the structural changes of the specific Charpy sample than the measured transition temperature values. From this point of view, it can be stated that they follow the neutron irradiation-generated material degradation very precisely. This is a highly 
important and serious conclusion of our work, which should be carefully checked in future research work.

Another important message of this work is that neutron irradiation-generated embrittlement highly depends on the initial microstructure state of the given material, and even different parts of the same reactor pressure vessel can behave in rather different ways. We have shown that the observed phenomenon is not due to the experimental implementation. We carefully analyzed the possible sources of uncertainty in our magnetic measurement (see Appendix A), and it was found that the scatter of the points due to possible measurement error is much less (see Figure A4) than the observed scatter of magnetic descriptors (see Figure 4). The error of the measurement is less than 1\%, while the scatter in the reference samples is around 5-6\%. Based on this analysis, only local material conditions can be responsible for the different embrittlement of material caused by the same dosage neutron irradiation.

On the other hand, even with the existence of a large amount of scatter of the points (as can be seen in Figure 5), the average correlation between the MAT parameters and the DBBT is more than satisfactory. If we want to estimate DBTT value for an unknown, irradiated, magnetically measured sample, we can use the experimentally determined correlation. By fitting a line on the points of Figure 5, as done in Figure 7, the fitted linear function is: DBTT $=47052 *$ MAT -849 .

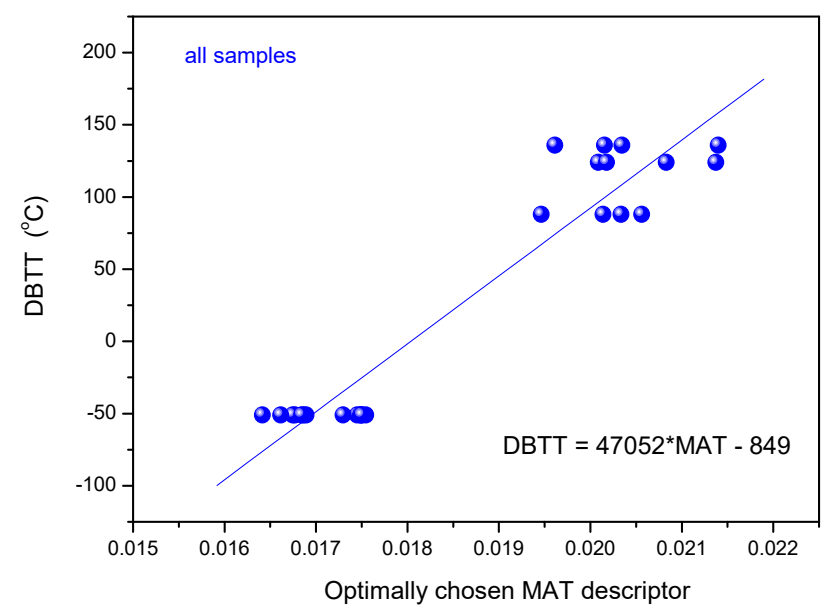

Figure 7. Estimation of the transition temperature from the MAT descriptors, measured on all investigated samples. The DBTT values are taken from the Charpy impact tests.

If the estimation is done based on measurements of magnetically selected samples, the fitted linear function is: DBTT $=46083^{*} \mathrm{MAT}-846$, which is remarkable close to the the one in Figure 7. In Figure 8, the linear fit from Figure 7 is also shown (blue dashed line) to illustrate the situation visually. This means that even a large scatter of points does not spoil the DBTT estimation significantly. We can use the found correlation for a reliable estimation of the transition temperature. Evidently, this numerical correlation cannot be generalized and it is not valid for different materials and conditions. Instead, these numbers are given only for a comparison in case of our present measurements. 


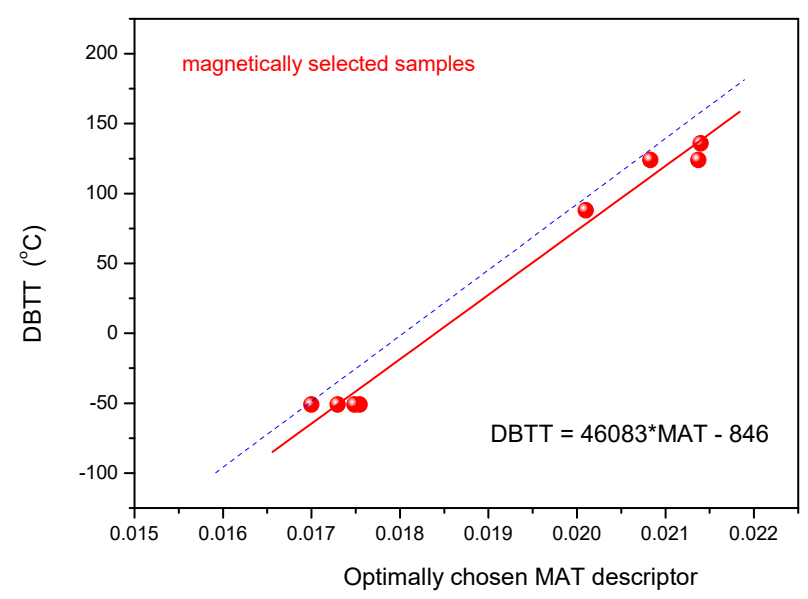

Figure 8. Estimation of the transition temperature from the MAT descriptors, measured on magnetically selected samples (subset). For a better comparison, the linear fit of Figure 7 is also shown as a blue dashed line.

\section{Conclusions}

A nondestructive magnetic method was applied on a reactor steel material for the inspection of neutron irradiation-generated embrittlement, and results were compared with the destructively measured transition temperature. A good and reliable correlation was found between these characteristics, which can be used for the estimation of DBTT (even in case of an unknown sample) from the results of magnetic measurement, in spite of the large scatter of points.

The reason of this large scatter was also analyzed, and it was attributed to the local material inhomogeneity, to the differences between their initial microstructure state before irradiation, and not to the measurement error. Furthermore, it has been clearly demonstrated by our experiments that, if the microstructure of the reference (not-irradiated) samples is close to each other-which is suspected based on the magnetic measurementsthe irradiation embrittlement can be characterized very well, with extremely low scatter of points by magnetic parameters.

Magnetically measured parameters seem to characterize more precisely the material embrittlement than the traditionally used destructive methods. In addition, the magnetic method can characterize the individual samples, contrary to some destructive methods, which can provide only statistical values. In the future, these facts can be a serious argument for the application of magnetic measurements in a reactor industry.

Based on the analysis of the measurement conditions, only local material conditions can be responsible for the different embrittlement of a material caused by the same dosage neutron irradiation.

In order to have similar results as for a Charpy (destructive tests) impact curve, a large variation of samples needs to be tested due to the inhomogeneity. However, the number of samples can be reduced because the fit with a selected number of samples in the average (middle) magnetic hysteresis loops gives similar results as for a large number of samples.

It was found that the neutron irradiation-generated embrittlement highly depended on the initial material characteristics; even the embrittlement of different parts of the same reactor steel block can be rather different. This result gives important additional information about the whole process of the neutron-generated embrittlement of reactor pressure steel. Our results can be useful for the future potential introduction of this (and in general, any) nondestructive evolution method.

Author Contributions: Conceptualization and original draft preparation: G.V.; investigation and methodology: G.V., I.S., and I.U.; project administration: A.G. All authors have read and agreed to the published version of the manuscript. 
Funding: This research was carried out in frame of the "NOMAD" project. This project (Nondestructive Evaluation System for the Inspection of Operation-Induced Material Degradation in Nuclear Power Plants) has received funding from the Euratom research and training programme 2014-2018 under grant agreement No 755330.

Institutional Review Board Statement: Not applicable.

Informed Consent Statement: Not applicable.

Data Availability Statement: The data are contained within the article.

Conflicts of Interest: The authors declare no conflict of interest.

\section{Appendix A. Analysis of Uncertainty of MAT Method}

There are three possible independent sources of error within the MAT measurements: electronic error, influence of not proper sample positioning, and uncertainty of matrix evaluation. All of these possible errors were analyzed.

\section{Appendix A.1. Electronic Error}

To see the influence of the possible electronic error, the permeability loops were measured several times in a row, without touching the sample/yoke system. As an illustration, the measured loops on the same sample (sample 1) are shown in Figure A1. It can be clearly seen on the graph that the loops are practically identical with each other; no difference can be found between them. The reproducibility of the measurement is almost $100 \%$; no influence of possible electronic noise is found. The same was experienced in all of the measured samples by analyzing the results of many measurements.

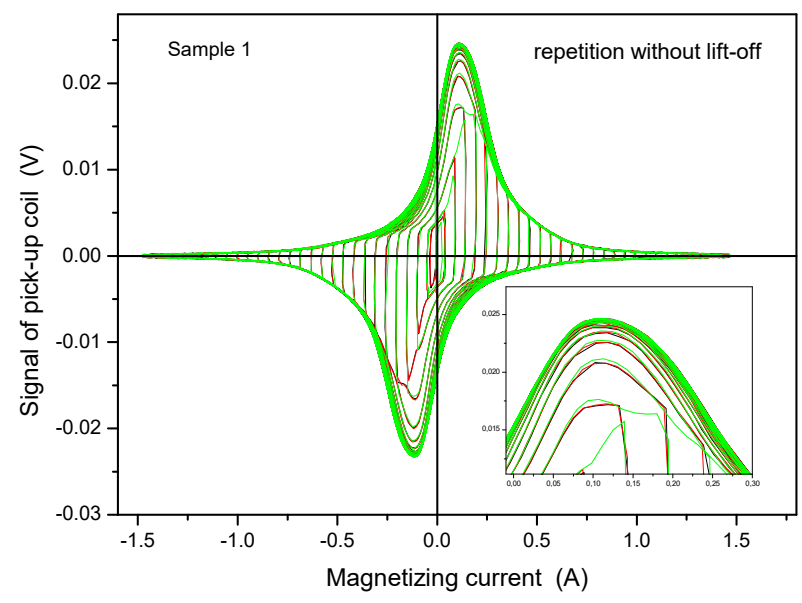

Figure A1. Series of permeability loops measured on sample 1, repeating the measurement after each other without replacing the sample. Insert: magnified part of the graph.

\section{Appendix A.2. Influence of Sample Repositioning}

The condition of the surface (possible dirt on the surface) or the different position of magnetizing yoke with respect to the sample surface can cause an error in the measured permeability loops. To check this possible source of error after repeating the measurements electronically (see above), the magnetizing yoke was removed from the sample surface, put back, and the measurement was repeated several times in a row. In this case, the repeatability of the measurement was also very good, although slightly worse than the previous case. The typical series of permeability loops after repositioning of magnetizing yoke are shown, again as an illustration, in Figure A2. The same was experienced in all of the measured samples by analyzing the results of many measurements. It can be stated that by using this procedure, the measurement error itself is also almost negligible, similarly to the electronic error. 


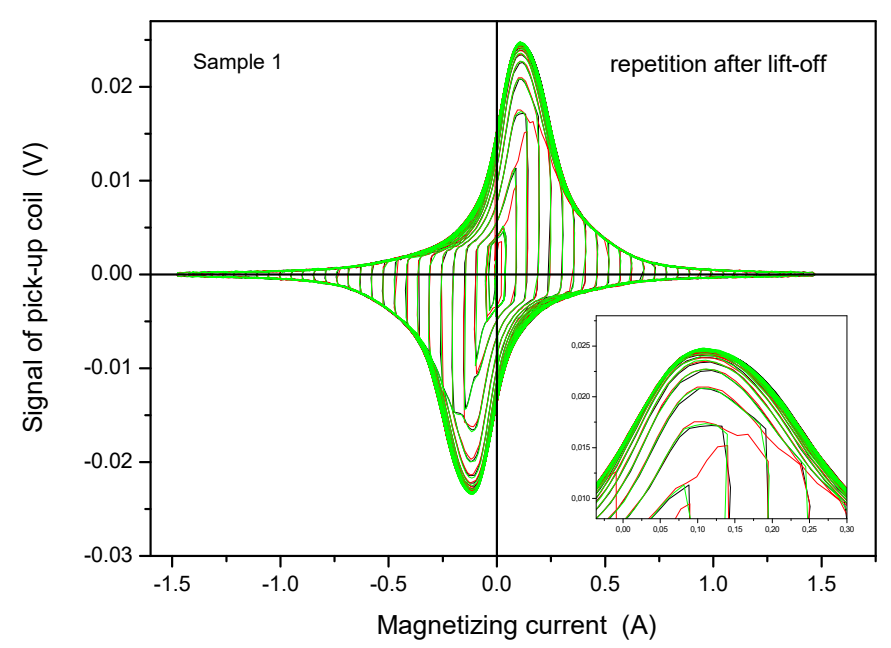

Figure A2. Series of permeability loops measured on sample 1 after reposition the magnetizing yoke. Insert: magnified part of the graph.

\section{Appendix A.3. MAT Evaluation of Permeability Loops}

During the evaluation, the permeability matrices of all samples were divided by the permeability matrices of reference (not-irradiated) sample(s). In this way, we obtained the correlation between the change of the magnetic parameters and the independent variable. Those parameters (MAT descriptors) were chosen from the big data pool, which resulted in the best correlation between the magnetic and independent parameters. Evidently, this process can also generate an error.

A sensitivity map helps a lot for choosing the optimal MAT descriptor. The relative sensitivity map of the $\mu$-degradation functions, $\mu(D B T T) \equiv \mu\left(h_{a}, h_{b}\right)$, is shown in Figure A3. The most sensitive MAT descriptors were taken from the top area of the "hills" (indicated by a red colour in Figure A3). The sensitivity map also indicates the reliability of the chosen descriptor. Big plateaus are favourable, where parameters only slightly depend on the actual choice of $h_{a}$ and $h_{b}$ field coordinates values. To minimize the error, we also performed a time check of all the neighbouring descriptors, and then we accepted the optimal MAT descriptor as satisfactory only if there were very small differences between the descriptors. This procedure also ensures that if the measurement is repeated (i.e., a long time after the first measurement), and if during the evaluation not the exact same magnetizing field $\left(h_{a}\right)$ and minor loop amplitude $\left(h_{b}\right)$ parameters are chosen, the error of the measurement remains small. This uncertainty of the MAT descriptor choice is the most significant error of the MAT measurement/evaluation, and it is considered as the real error of the measurement.

This process is shown for the investigated samples. The MAT descriptor, characterized by $h_{a}=-30 \mathrm{~mA}$ and $h_{b}=1080 \mathrm{~mA}$ field coordinate values, was found to give the best correlation between the MAT parameters and independent variable. In Figure A4, those descriptors are also shown, which were taken from the neighbouring positions $\left(h_{a}=-30 \mathrm{~mA}\right.$, $h_{b}=1080 \mathrm{~mA}$ ). Note that in this graph, the normalized MAT descriptors are given, because they are better in comparison in this case. It can be clearly seen that descriptors taken from this area are almost identical, and all of them properly express the correlation between the magnetic parameters and the transition temperature. 

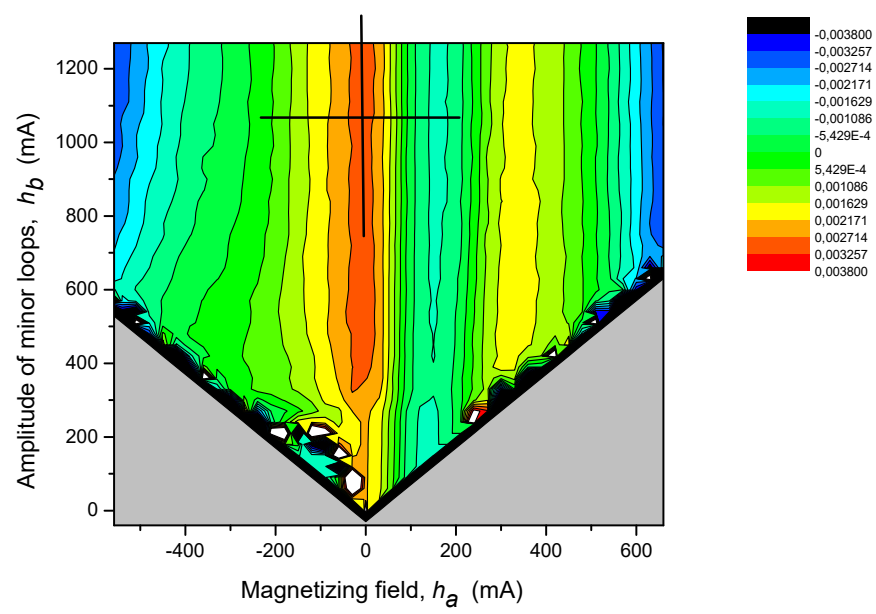

Figure A3. Relative sensitivity map of the $\mu$-degradation functions, $\mu$ (DBTT) $\equiv \mu\left(h_{a}, h_{b}\right)$. Crossing the lines indicates the $h_{a}=-30 \mathrm{~mA}$ and $h_{b}=1080 \mathrm{~mA}$ field coordinates of the $\mu_{i}(D B T T)$-degradation function presented in Figure 3.

Summing up of all possible sources of uncertainty, the error of the whole MAT evaluation is less than $1 \%$.

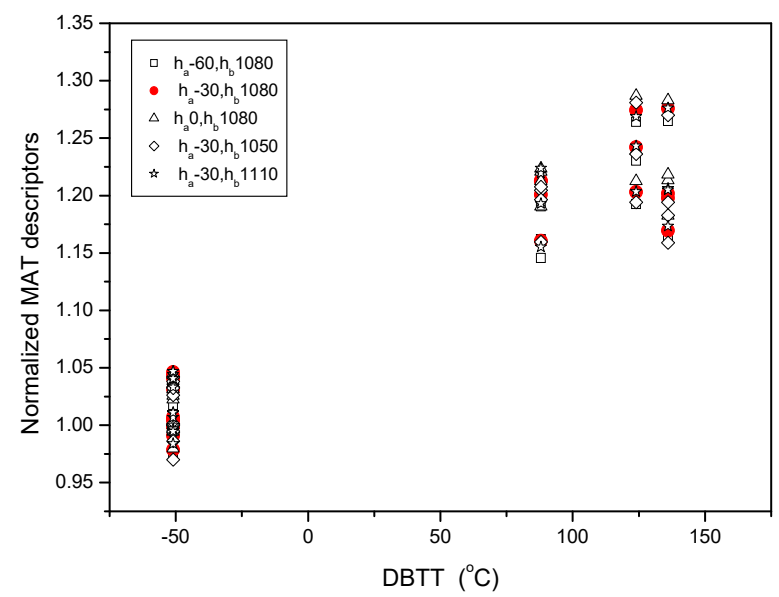

Figure A4. Neighbouring MAT descriptors (black open symbols) around the optimally chosen MAT descriptor (red full circle) as a function of the transition temperature.

\section{References}

1. Gillemot, F.; Barroso, S.P. Possibilities and difficulties of the NDE evaluation of irradiation degradation. In Proceedings of the 8th International Conference on Barkhausen Noise and Micromagnetic Testing (ICBM8), Kalpakkam, India, 11-12 February 2010; ISBN 978-952-67247-2-0.

2. Koutsky, J.; Kockk, J. Radiation Damage of Structural Materials; Elsevier Science Publishers: Amsterdam, The Netherlands, 1994.

3. Ferreño, D.; Gorrochategui, I.; Gutiérrez-Solana, F. Degradation due to neutron embrittlement of nuclear vessel steels: A critical review about the current experimental and analytical techniques to characterise the material, with particular emphasis on alternative methodologies. In Nuclear Power-Control, Reliability and Human Factors; Tsvetkov, P., Ed.; IntechOpen Limited: London, UK, 2011; ISBN 9789533075990. Available online: http:/ /www.intechopen.com/articles/show/title/non-destructivetesting-for-ageing-management-of-nuclear-power-components (accessed on 14 May 2020).

4. Niffenegger, M.; Leber, H.J. Monitoring the embrittlement of reactor pressure vessel steels by using the Seebeck coefficient. J. Nucl. Mater. 2009, 389, 62. [CrossRef]

5. Smith, R.L.; Rusbridge, K.L.; Reynolds, W.N.; Hudson, B. Ultrasonic attenuation, microstructure and ductile to brittle transition temperature in Fe-C alloys. Mater. Eval. 1993, 41, 219-222.

6. Jiles, D.C. Magnetic methods in nondestructive testing. In Encyclopedia of Materials Science and Technology; Buschow, K.H.J., Ed.; Elsevier Press: Oxford, UK, 2001; p. 6021. 
7. Dobmann, G.; Kern, R.; Altepeter, L.; Theiner, W. Quantitative hardening-depth-measurements up to $4 \mathrm{~mm}$ by means of micromagnetic microstructure multiparameter analysis (3MA): Review of Progress in Quantitative Nondestructive Evaluation, Williamsburg, Virginia (United States), 22-26 Jun. 1987. Vol. 7B, pp. 1471-1476. Edited by DD Thompson and DE Chimenti, Plenum Press, 1988. NDT E Int. 1991, 24, 44-46.

8. Barroso, S.P.; Horváth, M.; Horváth, Á. Magnetic measurements for evaluation of radiation damage on nuclear reactor materials. Nucl. Eng. Des. 2010, 240, 722-725. [CrossRef]

9. Dobmann, G.; Altpeter, I.; Kopp, M.; Rabung, M.; Hubschen, G. ND-materials characterization of neutron induced embrittlement in German nuclear reactor pressure vessel material by micromagnetic NDT techniques. In Electromagnetic Nondestructive Evaluation (XI); IOS Press: Amsterdam, The Netherlands, 2008; p. 54. ISBN 978-1-58603-896-0.

10. Kronmüller, H.; Fähnle, M. Micromagnetism and the Microstructure of Ferromagnetic Solids; Cambridge University Press: Cambridge, UK, 2003.

11. Vértesy, G.; Gasparics, A.; Uytdenhouwen, I.; Szenthe, I.; Gillemot, F.; Chaouadi, R. Nondestructive investigation of neutron irradiation generated structural changes of reactor steel material by magnetic hysteresis method. Metals 2020, 10, 642. [CrossRef]

12. Tomáš, I.; Vértesy, G.; Gillemot, F.; Székely, R. Nondestructive Magnetic Adaptive Testing of Nuclear Reactor Pressure Vessel Steel Degradation. J. Nucl. Mater. 2013, 432, 371-377. [CrossRef]

13. Rabung, M.; Kopp, M.; Gasparics, A.; Vértesy, G.; Szenthe, I.; Uytdenhouwen, I. Micromagnetic characterization of operation induced damage in Charpy specimens of RPV steels. Appl. Sci. 2021, 11, 2917. [CrossRef]

14. Tomáš, I. Non-destructive Magnetic Adaptive Testing of ferromagnetic materials. J. Magn. Magn. Mater. 2004, 268, 178-185. [CrossRef]

15. Tomáš, I.; Vértesy, G. Magnetic Adaptive Testing. In Nondestructive Testing Methods and New Applications; Omar, M., Ed.; IntechOpen: London, UK, 2021; ISBN 978-953-51-0108-6. Available online: http://www.intechopen.com/articles / show/title/ magnetic-adaptive-testing (accessed on 8 November 2020).

16. Uytdenhouwen, I.; Chaouadi, R. Non-destructive Evaluation (NDE) system for the inspection of operation-induced material degradation in nuclear power plants-Overview of the neutron irradiation campaigns. In ASME 2020Pressure vessels EPiping Conference; Non_Destructive Examination, ASME: New York, NY, USA, 2020; Volume 7.

17. DeVan, M.J.; Lowe, A.L.; Hall, J.B. Fracture toughness test results of thermal aged reactor vessel materials. ASTM Spec. Tech. Publ. 1996, 1270, 660-669. 